\title{
No Increased Bone Formation around Alendronate or Omeprazole Loaded Bioactive Bone Cements in a Femoral Defect
}

\author{
ESTHER W.H. BODDE, M.D., ${ }^{1}$ RICK S.Z. KOWALSKI, Ph.D., ${ }^{2}$ \\ PAUL H.M. SPAUWEN, M.D., Ph.D., ${ }^{3}$ and JOHN A. JANSEN, D.D.S., Ph.D. ${ }^{1}$
}

\begin{abstract}
Alendronate and omeprazole have been found to influence bone healing by interfering with osteoclastic activity, resulting in increased bone formation. The biological effect of these conventional drugs, incorporated into bioactive bone cement (G2B1), was investigated in a rabbit model. The 2 materials and a control were inserted in defects created in the femoral condyle of rabbits. Implantation time was 6 and 12 weeks. After retrieval, micro-computed tomography and histomorphometry were performed to quantify bone mineral density (BMD) and bone volume (BV) of the implant-surrounding bone mass and the percentage of bone-to-implant contact. BMD and BV were similar in all groups. The percentage of bone-to-implant contact was significantly lower in the alendronate and omeprazole groups than in controls after 6 weeks of implantation. After 12 weeks, this difference in bone contact disappeared for the omeprazole but not for the alendronate implants, which were almost completely surrounded by a fibrous capsule, associated with a limited inflammatory response. In conclusion, in the current study, alendronate and omeprazole did not result in better bone healing when incorporated into bioactive bone cement than did plain control implants. Moreover, an additional cytotoxicity assay revealed that alendronate evoked a toxic response.
\end{abstract}

\section{INTRODUCTION}

B ONE REMODELING IS based on the existence of a delicate equilibrium between bone deposition by osteoblasts and bone resorption by osteoclasts. ${ }^{1-3}$ The regulation of this complex process is dependent on the effect of local and systemic factors on mesenchymal cells of the osteoblastic lineage and hemopoietic precursors to form osteoclasts. ${ }^{4}$ An increase in bone formation can be achieved by influencing 1 of the 2 mechanisms: bone formation or bone resorption. Such an approach can be used for targeted therapeutic interventions in bone diseases, which are associated with bone loss, or for improvement in the bioactivity of biomaterials used for bone replacement.

In view of this, bone cements can be applied as grafting materials to obtain fracture stability or for the fixation of prostheses. $^{5-7}$ A strategy to improve the bone regenerative properties of bone cements can be the incorporation of biological active agents. A well-known example of this approach is scaffolds provided with bone growth stimulating factors, ${ }^{8,9}$ but the high costs of these growth factors limit their wide clinical application. The use of relatively inexpensive conventional pharmaceuticals, which can have a local effect on the bone remodeling process, might therefore be an alternative option. A class of pharmaceuticals is available, including bisphosphonates and proton pump inhibitors (PPIs), that have been found to interfere with osteoclastic activity, inducing an increase in local bone density. Osteoclasts resorb bone mineral because they make an acidic extracellular environment in their ruffled border zone through the action of so-called proton pumps.

\footnotetext{
${ }^{1}$ Department of Periodontology and Biomaterials, Radboud University Nijmegen Medical Center, Nijmegen, The Netherlands.

${ }^{2}$ DePuy CMW, Marton, Blackpool, England.

${ }^{3}$ Department of Plastic and Reconstructive Surgery, Radboud University Nijmegen Medical Center, Nijmegen, The Netherlands.
} 
Nitrogen-containing bisphosphonates such as alendronate hamper osteoclastic bone resorption by inhibiting the key enzyme farnesyl pyrophosphate synthetase, leading to disruption of the organization of the cytoskeleton, which causes inactivity and apoptosis of osteoclasts. ${ }^{10}$ These medicines are administered systemically to treat various diseases associated with excessive bone resorption, including Paget's disease, myeloma, bone metastases, and osteoporosis. ${ }^{11}$ However locally applied bisphosphonates have also been shown to influence bone resorption. ${ }^{12-18}$ In addition to the use of bisphosphonates, the activity of the proton pumps can be directly targeted. Omeprazole is a gastric PPI used for the treatment of acid-related diseases, including gastroesophageal reflux and peptic ulcer diseases. ${ }^{19}$ In addition to the inhibition of gastric parietal cell membrane $\mathrm{H}+$, $\mathrm{K}(+)$-adenosine triphosphatase (ATPase), it is suggested that this drug also inhibits the vacuolar-type $\mathrm{H}(+)$-ATPase found in osteoclasts. ${ }^{20-24}$ These vacuolar proton pumps play a role in the formation of an acidic environment in the extracellular ruffled border zone of osteoclasts, which is crucial for bone resorption. $^{25,26}$

The present study examined whether the incorporation of a bisphosphonate (alendronate) or a PPI (omeprazole) into bioactive bone cement could result in an increase in local bone density and bone-to-implant contact through inhibition of osteoclast activity.

\section{MATERIALS AND METHODS}

\section{Materials}

Bioactive bone cement that had previously been shown to be biocompatible and osteoconductive ${ }^{27}$ was used as a carrier material for the pharmaceuticals. DePuy CMW (Blackpool, England) supplied bone cement (G2B1) composed of $\beta$ tricalcium-phosphate (TCP) particles; methylmethacrylatemethylacrylate copolymers as the powder components; and methylmethacrylate, urethane dimethacrylate, and tetrahydrofurfuryl methacrylate as the liquid components. ${ }^{27}$ Three types of implants were prepared out of this material $(\mathrm{I}=$ plain $\mathrm{G} 2 \mathrm{~B} 1, \mathrm{II}=\mathrm{G} 2 \mathrm{~B} 1$ with alendronate, and $\mathrm{III}=\mathrm{G} 2 \mathrm{~B} 1$ with omeprazole). Sodium alendronate or omeprazole was mixed through the cement powder in a ratio of $4.76 \%$, then $20 \mathrm{~g}$ of cement powder was mixed with $11.25 \mathrm{~g}$ of cement liquid to create bone cement rods with a diameter of $5.1 \mathrm{~mm}$ and a length of $8 \mathrm{~mm}$. The total quantity for both types of drugs in the cement was $3.05 \%$ by weight $(\sim 8.8 \mathrm{mg}$ per implant). The material was sterilized using gamma radiation (Gammastar, Wageningen, the Netherlands).

\section{Surgical procedure}

Twenty-four healthy 7-month-old female New Zealand White rabbits weighing $3976 \pm 332 \mathrm{~g}$ were included as experimental animals in this study. National guidelines for the care and use of laboratory animals were observed. The Experimental Animal Committee of the Radboud University reviewed and approved the research.

The operation was performed under general inhalation anesthesia. The anesthesia was induced using an intravenous injection of Hypnorm $(0.315 \mathrm{mg} / \mathrm{mL}$ fentanyl citrate and $10 \mathrm{mg} / \mathrm{mL}$ fluanisone) (Janssen Pharmaceutica, Beerse, Belgium) and atropine and maintained using a mixture of nitrous oxide, isoflurane, and oxygen through a constant volume ventilator. The rabbits were connected to a heart monitor. To reduce perioperative infection risk, antibiotic prophylaxis was given (enrofloxacin 2.5\%, 5-10 mg/kg; Bayer Healthcare, Mijdrecht, the Netherlands).

The rabbits were immobilized on their backs and both hind limbs were shaved, washed, and disinfected with chlorhexidine. The femoral condyles were exposed through a medial longitudinal incision. Trabecular bone defects (5.1 $\mathrm{mm}$ in diameter and $8 \mathrm{~mm}$ in depth) were created in both limbs under continuous saline cooling using a dental bur (Elcomed 100, W\&H Dentalwerk Buermoos GmbH, Buermoos, Austria). First a 2.3-mm drill bit (Twist TriSpade drill, DRIVA, Implacom BV, Garderen, The Netherlands) was used as a pilot. Then the defect was gradually enlarged until a final defect size of $5.1 \mathrm{~mm}$ was created. All bits were fashioned with an 8-mm stop to ensure a defect of precisely $8 \mathrm{~mm}$ in depth. Debris was removed using irrigation with saline solution. A polymeric implant was placed into each trabecular bone defect. Subsequently, the wound was closed in layers using resorbable Vicryl 4-0 suture material (Johnson \& Johnson, St.Stevens-Woluwe, Belgium). Implantation time was 6 or 12 weeks. The animals were randomly assigned to 1 of the 3 groups ( $n=8$ for each implant and time period).

To minimize post-operative discomfort, Finadyne (Schering-Plough, Segre, France) $(1 \mathrm{mg} / \mathrm{kg})$ was administered intramuscularly peroperatively and was continued for 2 days after surgery. Some question about the use of this non-steroidal anti-inflammatory drug in bone surgery exists, because bone repair could possibly be impaired, ${ }^{28}$ but as in humans, these drugs are a standardized postoperative regimen for pain relief, ${ }^{29}$ so administration in our experimental animals was considered to be justified. The animals were housed in conventional rabbit cages, which allowed for unrestricted weight-bearing activity and were observed for signs of pain, infection, and proper activity. At the end of the implantation periods, the rabbits were killed using an intravenous injection of pentobarbital (CEVA Sante Animale BV, Libourne, France), and implants with surrounding tissue were retrieved for micro-computed tomography $(\mu \mathrm{CT})$ and histological evaluation.

\section{Micro-computed tomography}

After retrieval, the femoral condyles were fixed in phosphate-buffered formaldehyde solution ( $\mathrm{pH} 7.4)$ and dehydrated in ethanol $70 \%$. Three-dimensional $\mu \mathrm{CT}$ images 
were made to analyze the bone mineral density (BMD) and bone volume (BV) of the implant-surrounding bone mass. The specimens were wrapped in Parafilm M (Pechiney Plastic Packaging, Chicago, IL) to prevent drying during scanning. Then all samples were scanned at an energy of $101 \mathrm{kV}$ and intensity of $96 \mu \mathrm{A}$ with a resolution of $37.41 \mu \mathrm{m}$ pixel using an aluminum filter $(1 \mathrm{~mm})$ (Skyscan-1072 X-ray micro-tomograph, TomoNT version 3N.5, Skyscan, Aartselaar, Belgium). In addition, calibration rods with standardized BMD were scanned as reference. Cone-Beam reconstruction (version 2.15, Skyscan) was performed. All scan and reconstruction parameters applied were identical for all specimens and calibration rods.

The data were analyzed using a CT analyser (version 1.4, Skyscan). The region of interest (ROI) was specified as an annular area with a diameter of $0.85 \mathrm{~mm}$ surrounding the implants over a length of $3 \mathrm{~mm}$ (Fig. 1) covering similar areas located in trabecular bone and a small part protruding in the medullary cavity. In this area, BMD and BV were determined. BMD is defined as the amount of bone mineral per unit volume of bone tissue $\left(\mathrm{g} / \mathrm{cm}^{3}\right)$. It was calibrated using calibration rods with known BMD $\left(0.25 \mathrm{~g} / \mathrm{cm}^{3}\right.$ and $0.75 \mathrm{~g} / \mathrm{cm}^{3}$ ) and a Hounsfield Unit calibration to water and air density. As advised by Skyscan, the mean (total) value for density, which is an average of trabecular bone and bone marrow, was used to represent the BMD of the trabecular bone surrounding the implants. In addition, BV $\left(\mathrm{mm}^{3}\right)$ was expressed as a percentage of the total ROI volume.

\section{Histological procedures}

After $\mu \mathrm{CT}$ scanning, the condyles were cut into smaller specimens suitable for histological processing. The specimens were dehydrated in increasing ethanol concentrations (70-100\%), treated with acetone for $6 \mathrm{~h}$, and embedded (non-decalcified) in modified methylmethacrylate (MMA) consisting of a mixture of $300 \mathrm{~mL}$ of MMA, $30 \mathrm{~mL}$ of dibutylphthalate, and $5 \mathrm{~g}$ of 2,2'azabisisobutyronitrile $98 \%$.
After polymerization, thin sections $(7 \mu \mathrm{m})$ were cut in a direction parallel to the longitudinal axis of the implant using a rotary microtome with semi-motorized specimen feed and profile D disposable blades (Leica RM 2245, Wetzlar, Germany). The sections were stretched on $3 \%$ gelatincoated slides, dried for 48 hat $37^{\circ} \mathrm{C}$ in a slide press, and stained with tartrate-resistant acid phosphatase (TRAP). To perform TRAP staining, the sections were first deplastified with chloroform and xylene and rinsed in decreasing ethanol solutions and distilled water. Then the deplastified sections were transferred into 0.2-M TRIS-magnesium chloride $\left(\mathrm{MgCl}_{2}\right)$ buffer $(\mathrm{pH} 9.0)$ for $2 \mathrm{~h}$ at $37^{\circ} \mathrm{C}$. After rinsing with distilled water, the sections were incubated in a solution consisting of hexazotized pararosaniline $(4 \mathrm{~mL})$, naphthol AS-BI phosphate $(25 \mathrm{mg}), \mathrm{N}, \mathrm{N}$-dimethylformamide $(2.5 \mathrm{~mL})$, veronal buffer $(12.5 \mathrm{~mL})$, and $\mathrm{MgCl}_{2}(0.5 \mathrm{~g})(\mathrm{pH}$ 5.0) at $37^{\circ} \mathrm{C}$. Positive staining developed within $30 \mathrm{~min}$. Positive control sections were included.

Additional thicker sections $(15-20 \mu \mathrm{m})$ were prepared in a similar way using a modified sawing microtome technique. These sections were stained with methylene blue and basic fuchsin and were used for histological evaluation and histomorphometrical analysis.

\section{Histomorphometrical analysis}

All sections were quantitatively scored for the percentage of bone-to-implant contact using computer-based image analysis techniques (Leica Qwin Pro-image analysis system, Wetzlar, Germany) and blinded for the reviewer. From a transverse overview of the defect, 6 identical areas were digitalized (magnitude 10x) (Fig. 2). In these regions, the amount of bone contact was determined, defined as the length at which direct bone-to-implant contact was seen without an intervening soft tissue layer. Bone contact was then expressed as a percentage of the sum of bone contact in the 6 areas to the entire implant length of these areas. The measurements of 3 sections for each sample were averaged.
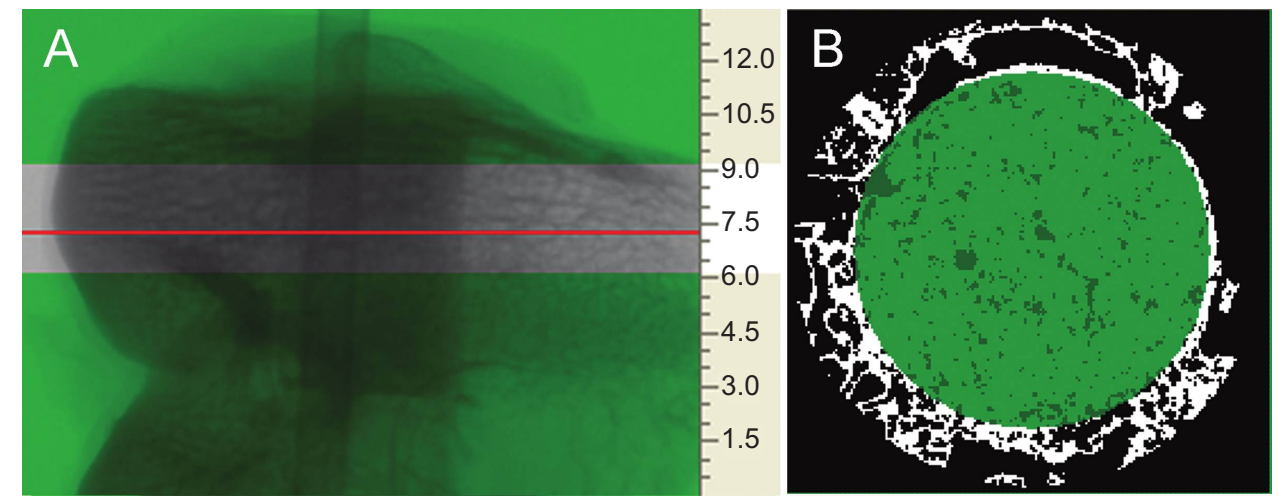

FIG. 1. The region of interest as specified with computed tomography (CT) analyzer shown in a (A) longitudinal and (B) transverse micro-CT image. Color images available online at www.liebertpub.com/ten. 


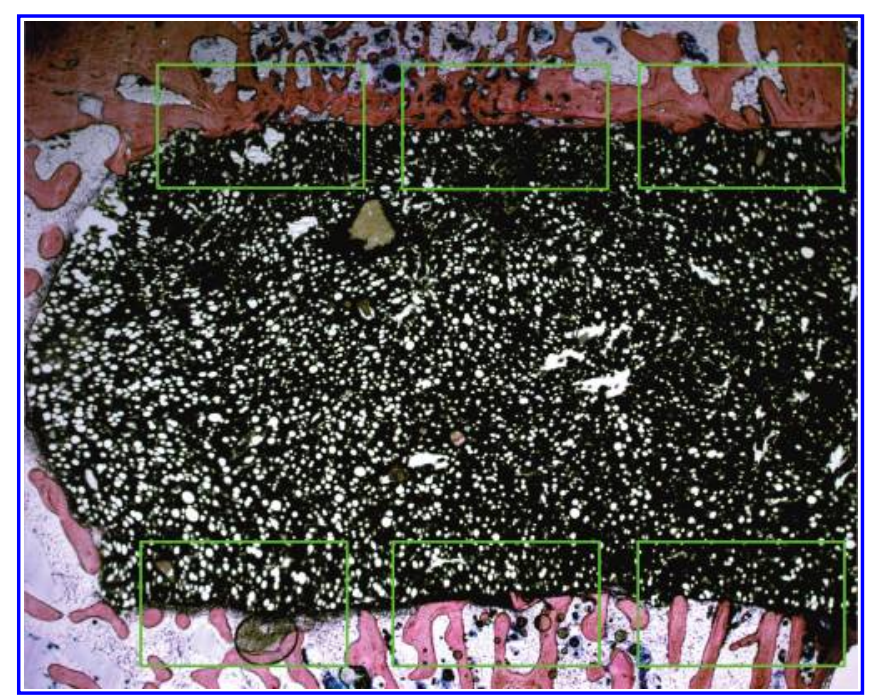

FIG. 2. Bone-to-implant contact was determined using histomorphometrical analysis in 6 identical areas covering the whole implant surface. Color images available online at www.liebertpub .com/ten.

\section{Statistics}

All statistical analyses were performed with GraphPad Instat 3.05 software (GraphPad Software Inc, San Diego, CA), using a 1-way analysis of variance (ANOVA) and unpaired $t$-test with Welch correction. Differences were considered significant at $p$-values less than 0.05 .

\section{RESULTS}

\section{Macroscopical evaluation}

All rabbits endured the surgical procedure well and remained in good health during the experimental periods. At sacrifice, no signs of inflammation or adverse tissue reaction were seen. Post-sacrifice radiographs as obtained with $\mu \mathrm{CT}$ imaging revealed that the implants were located mostly in trabecular bone (Fig. 3) and that this trabecular area was similar between the different samples.

\section{Micro-computed tomography}

Three-dimensional $\mu \mathrm{CT}$ images were created to analyze the BMD and BV of the implant-surrounding bone mass. The results of the measurements are listed in Table 1. No significant difference in BMD was found between the implant types after $6(p=0.12)$ or 12 weeks of implantation $(p=0.31)$ (ANOVA). In addition, no significant difference in BMD was seen between the 6- and 12-week groups (unpaired $t$-test with Welch correction). The percentage of $\mathrm{BV}$ in the ROI as determined using $\mu \mathrm{CT}$ was not significantly different between the implant types after $6(p=$ $0.18)$ and 12 weeks of implantation $(p=0.42)$ (ANOVA). Comparing 6 and 12 weeks of implantation time, alendronate implants showed a trend $(p=0.07)$ toward lower BV after 12 weeks. In the omeprazole implants, BV was significantly ( $p=0.008$ ) lower after 12 weeks implantation than after 6 weeks (unpaired $t$-test with Welch correction).

\section{Descriptive light microscopy}

Implantation time 6 weeks. The implant material was clearly visible in the histological sections, showing $\beta$-TCP particles embedded in the polymeric matrix. In all specimens, the implants were found to be inserted and surrounded by trabecular bone.

The plain control material was never associated with an inflammatory response (Fig. 4A). The surface of the plain control material was partly covered with a layer of bone. Occasionally, new bone formation was associated with the presence of osteoid lined with osteoblasts. There was tight contact between the new bone and plain control surface

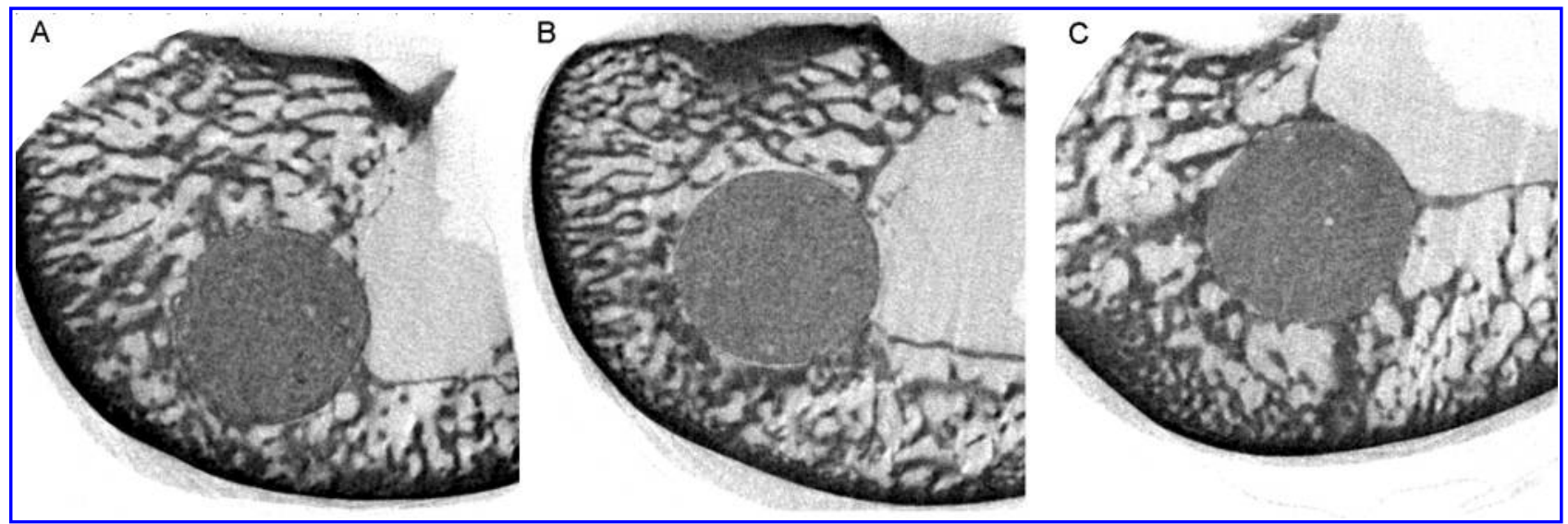

FIG. 3. Post-sacrifice radiographs as obtained with micor-computed tomography imaging revealed that the implants were mainly located in trabecular bone: (A) plain control, (B) alendronate, and (C) omeprazole implant. 
Table 1. The Bone Mineral Density (BMd) and Percentage of Bone Volume (BV) in the Region of Interest As Determined Using Micro-Computed Tomography

\begin{tabular}{|c|c|c|c|c|}
\hline & \multicolumn{2}{|c|}{$B M D\left(\mathrm{~g} / \mathrm{cm}^{3}\right)$} & \multicolumn{2}{|c|}{$B V(\%)$} \\
\hline & 6 weeks & 12 weeks & 6 weeks & 12 weeks \\
\hline & \multicolumn{4}{|c|}{ Mean \pm Standard Deviation } \\
\hline Plain & $0.71 \pm 0.03$ & $0.74 \pm 0.07$ & $15.5 \pm 2.6$ & $13.7 \pm 2.7$ \\
\hline Alendronate & $0.76 \pm 0.06$ & $0.76 \pm 0.06$ & $18.3 \pm 3.9$ & $14.8 \pm 2.6$ \\
\hline Omeprazole & $0.74 \pm 0.06$ & $0.71 \pm 0.06$ & $18.5 \pm 3.9$ & $13.3 \pm 1.6^{\dagger}$ \\
\hline
\end{tabular}

No significant differences were found in BMD between the groups. BV was significantly $\left(^{\dagger}\right)$ lower after 12 than after 6 weeks of implantation in the omeprazole implants.

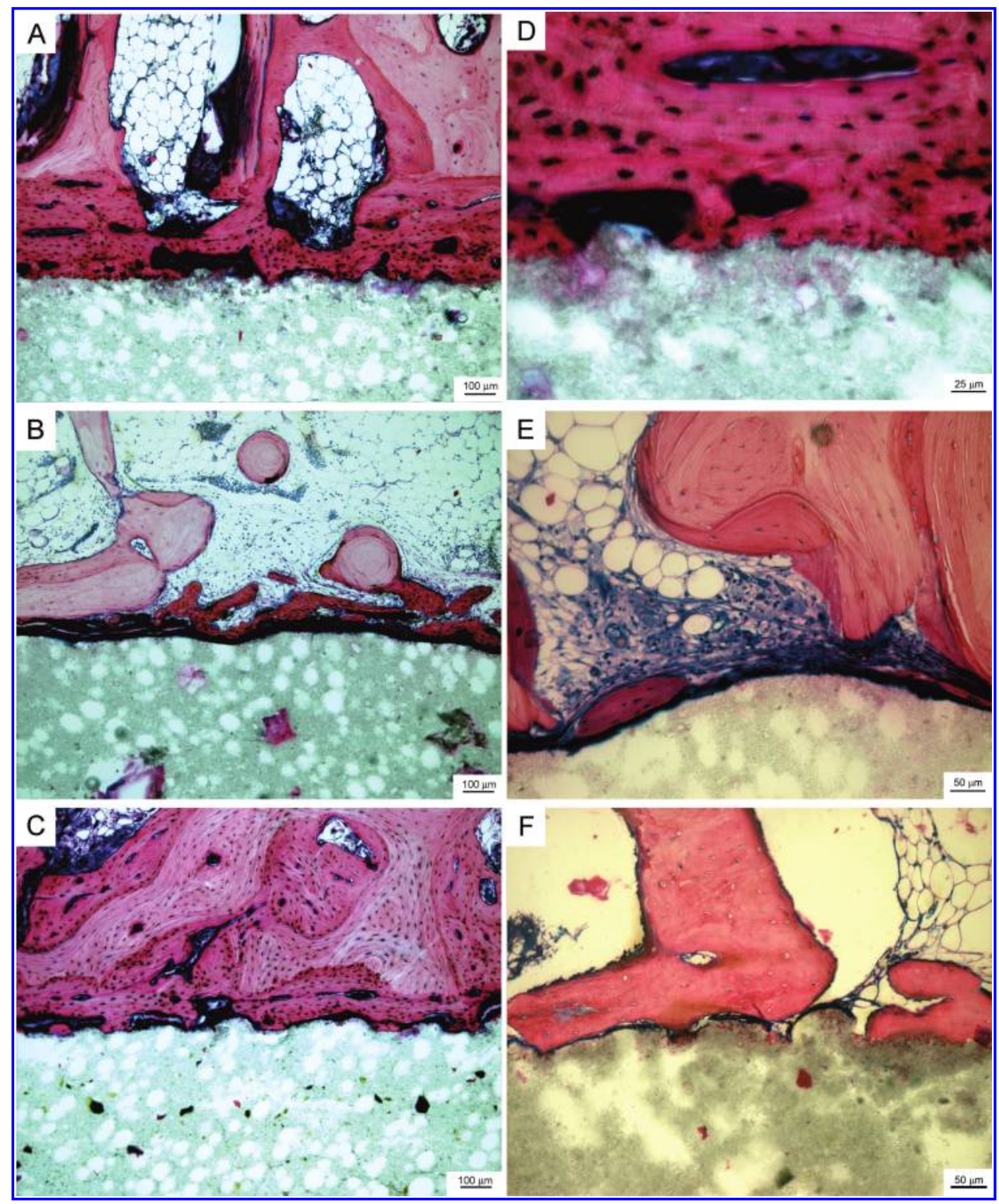

FIG. 4. Histological sections after 6 weeks of implantation of (A) plain, (B) alendronate, and (C) omeprazole implants (higher magnifications in (D, E, and F)). Methylene blue and basic fuchsin staining. Color images available online at www.liebertpub.com/ten. 
(Fig. 4D). No intervening fibrous tissue layer was present. At trabecular voids and in areas where no bone contact was seen, the implant surface was in direct contact with bone marrow. No clear fibrous capsule was visible in these areas.

The alendronate and omeprazole implants, on the other hand, showed a completely different bone response (Fig. $4 \mathrm{~B}, \mathrm{C})$. These materials were mostly surrounded with a fibrous tissue capsule, and only limited direct bone-implant contact was observed (Fig. 4E, F). Fibrous tissue capsule formation was associated with an inflammatory response in trabecular void areas (Fig. 4E).

Implantation time 12 weeks. At 12 weeks of implantation, bone response to the plain control implants was similar to that at 6 weeks. Bone remodeling had continued, and bone appeared to be somewhat more mature. Between bone and implant, close contact was still present (Fig. 5A). Moreover, bone was integrated into the superficial layer of the material, indicating degradation of the outer implant surface. Intermittently, resorption lacunae were present, characterized by voids filled with osteoclast-like cells (Fig. 5A).

A fibrous tissue capsule, associated with a limited inflammatory response, almost completely surrounded the alendronate implants (Fig. 5B).

The bone response to the omeprazole implants was different at 6 weeks of implantation. Significantly more bone was observed in tight contact with the implant surface (Fig. 5C). In areas of tight contact, degradation of the outer implant surface was seen. In areas not covered by bone, a thin fibrous capsule still surrounded the implants, although capsule formation was no longer associated with an inflammatory response.

TRAP staining. In all sections (i.e., for all implant formulations and both implantation times), TRAP-positive cells were present in the surrounding trabecular bone and at the bone-implant interface (Fig. 6). TRAP staining was not found to be specific to differentiate between osteoclasts, macrophages, and giant cells. In addition, the aspecific morphology of these interfacial cells excluded their proper characterization. As a consequence, no correct quantification was possible. Nevertheless, the number of TRAP-positive cells was limited in the close vicinity of the implants, and no clear difference between the 3 implant materials was seen.

\section{Histomorphometry}

Results of the bone-to-implant contact measurements are presented in Table 2. Bone contact was significantly ( $p=$ 0.001 ) lower in the alendronate and omeprazole groups than in the plain control implants after 6 weeks of implantation (ANOVA). In the 12-week group, alendronate implants had significantly $(p<0.001$, ANOVA) lower bone contact than plain and omeprazole implants. Comparing 6 and 12 weeks implantation time, alendronate implants
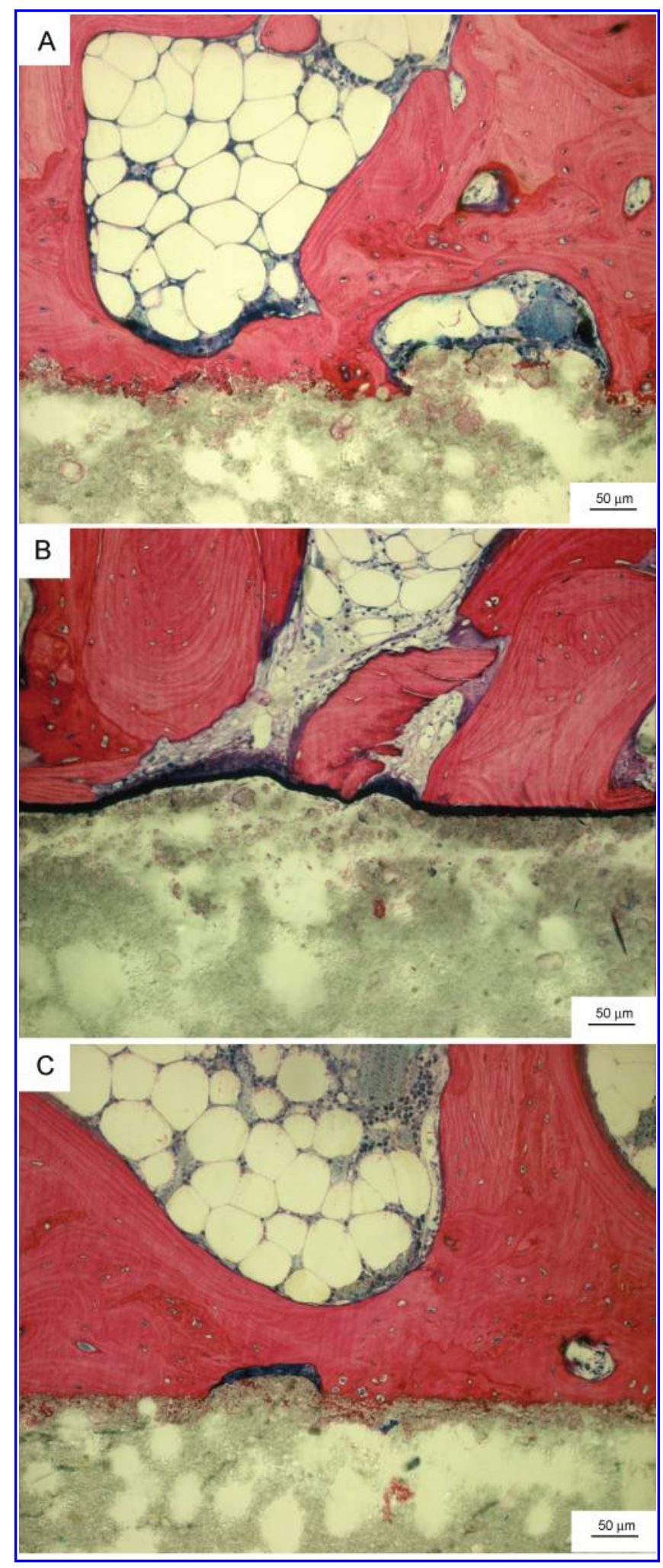

FIG. 5. Histological sections after 12 weeks of implantation of (A) plain, (B) alendronate, and (C) omeprazole implants. Methylene blue and basic fuchsin staining. Color images available online at www.liebertpub.com/ten. 


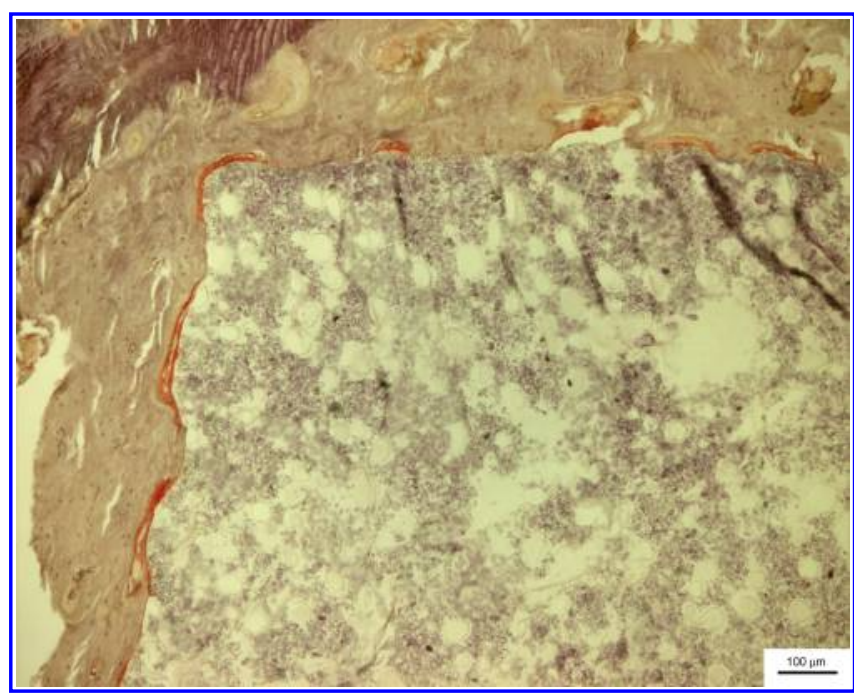

FIG. 6. Tartrate-resistant acid phosphatase-positive cells at the interface of a plain control implant after 6 weeks of implantation. Color images available online at www.liebertpub.com/ten.

showed a trend ( $p=0.07)$ toward less bone contact after 12 weeks, whereas in the omeprazole implants, the bone contact increased significantly over time $(p=0.008)$ (unpaired $t$-test with Welch correction).

\section{DISCUSSION}

The local application of bone antiresorptive drugs is an interesting approach to enhance the bone regenerative behavior of bone-replacing materials, compared, for instance, with expensive growth factor inclusion. In the present study, the bisphosphonate alendronate and the gastric proton pump inhibitor omeprazole were incorporated into bioactive bone cement and examined for their positive effect on bone healing, but neither drug improved bone healing over plain control implants.

One possible indication for the composite bone cement (G2B1) is for percutaneous transpedicular vertebroplasty; a

Table 2. The Percentage of Bone-to-Implant Contact As Determined Using Histomorphometry

\begin{tabular}{lcc}
\hline & Bone contact $(\%)$ Mean \pm Standard Deviation \\
\cline { 2 - 3 } & 6 weeks & 12 weeks \\
\hline Plain & $37.6 \pm 10.1^{*}$ & $36.1 \pm 18.4$ \\
Alendronate & $11.9 \pm 10.6$ & $3.7 \pm 4.7^{* *}$ \\
Omeprazole & $19.6 \pm 14.6$ & $39.3 \pm 9.4^{\dagger}$ \\
\hline
\end{tabular}

In the 6-week group, bone contact was significantly $(*)$ higher in the plain implants than in the alendronate and omeprazole groups. After 12 weeks, the alendronate implants showed significantly $(* *)$ less bone contact than the other groups. In the omeprazole implants, the bone contact increased significantly over time $\left(^{\dagger}\right)$. previous animal study showed the biocompatible and osteoconductive properties of the material. ${ }^{27}$ The present study confirmed this finding, because a reasonable amount of new bone was tightly attached to the plain control implants after 6 weeks of implantation, which remained at 12 weeks. Apparently, the inclusion of $\beta$-TCP particles improves the bone response better than exclusively polymethylmethacrylatebased materials, which is consistent with previous investigations studying calcium phosphate-based bone cements. ${ }^{30-33}$ In percutaneous transpedicular vertebroplasty, but also in other orthopedic and dental procedures, it is important to obtain optimal binding between bone and implanted material. The inclusion of bone antiresorptive agents might contribute to faster and enhanced ingrowth of new bone into bone cement composites with greater bone density in the implant surroundings. Topical application of these bonestimulating agents might be preferable to a systemic route, because negative side effects at locations remote from the defect area might be prevented.

The current study had several limitations. First, only one dose of alendronate and omeprazole was incorporated into the bone cement. The method of inclusion and the dosage were based on previous studies by other investigators, who proved that the inclusion of 3 to $4 \mathrm{wt} \%$ of gentamycin and alendronate did not affect the mechanical or setting properties of acrylic cement. ${ }^{34,35}$ Second, the elution of the included pharmaceuticals from the cured cement was not investigated. This was decided because other studies in our laboratory confirmed that in vitro release assays provide completely different results from the in vivo situation, ${ }^{36}$ and in our laboratory, no method was available to determine the in vivo release of alendronate and omeprazole. However, we suppose that the mechanism of elution of both pharmaceuticals will be similar to the elution of antibiotics from an antibiotic-loaded acrylic bone cement (i.e., a surface phenomenon). ${ }^{37}$ Third, no assays were performed to verify the mode of action and cellular uptake of the released alendronate and omeprazole. Such tests were out of the scope of the current study, in which we only wanted to prove the occurrence of a clinical effect of local delivery of both pharmaceuticals by inclusion in bone cement. Nevertheless, we must emphasize that measures were taken to maintain the bone cement at $37^{\circ} \mathrm{C}$ to $40^{\circ} \mathrm{C}$ during the curing process in order not to hamper the activity of the alendronate and omeprazole.

Alendronate, as a nitrogen-containing bisphosphonate, is known to inhibit osteoclastic bone resorption by inhibition of a key enzyme of the mevalonate pathway when administered systemically. ${ }^{10}$ In this way, the lack of this enzyme will dominate the bone repair equilibrium of bone resorption and formation. It can be hypothesized that the topical application of alendronate will modify the local osteoclastic activity and thereby slow down the bone resorption during initial remodeling, ${ }^{12}$ leading to better bone formation around the implant, although in the present study, topically applied alendronate did not enhance the local bone conditions around 
G2B1 cement or the bone-to-implant contact more than did the plain control samples. Moreover, a negative response was seen, because the contact between cement and bone was significantly lower than with the control material, and a fibrous capsule associated with an inflammatory infiltrate mainly covered the cement surface. Nevertheless, bisphosphonates have been shown to affect bone formation in several studies when applied locally. This included greater bone contact, peri-implant $\mathrm{BV}$ or density fractions, and biomechanical implant fixation in rat $^{16,38-40}$ and canine models. ${ }^{13-15,17,18}$ Similar results in osteoconduction and alveolar bone repair were found between loaded and unloaded bisphosphonate-hydroxyapatite implants placed in root sockets of goats and humans. ${ }^{41}$ An explanation for the limited bone response as found in the current study might be too-high doses of alendronate being used in combination with the mode of delivery. Unfortunately, a direct comparison between the different studies is not possible because of dissimilar animal species and study design, such as mode of delivery. Peter et al., ${ }^{16}$ who revealed that intermediate doses of zoledronate achieved the greatest mechanical fixation of hyaluronic acid-coated titanium implants placed in rat condyles, showed a dose-dependent effect of locally released bisphosphonate. It has been suggested that high doses of alendronate impair the bone repair process by uncoupling the balanced osteoclastic and osteoblastic activity ${ }^{42,43}$ or increasing local acidity and thereby interfering with antiresorptive activity. ${ }^{39}$ In addition, non-therapeutic doses of alendronate can cause a toxic reaction, ${ }^{44}$ which the formation of a fibrous tissue capsule and inflammatory response supports. Therefore, we performed an additional cytotoxicity assay with materials prepared in the same way as used in the animal study to verify this suggestion. This test revealed cell death of fibroblasts surrounding alendronate specimens after $72 \mathrm{~h}$ of incubation (Fig. 7B), similar to a negative control of brass. In contrast, confluent fibroblasts in close contact to the material were found around plain and omeprazole specimens (Fig. 7A/C). Although fibroblastic cell death can also be related to the working mechanism of alendronate, as was seen for gastrointestinal epithelial cells, $^{45}$ it must be considered that the sample formulations used in the present study had a noxious effect.

An additional explanation for impaired bone healing after bisphosphonate treatment involves a clinical problem that has remained largely unexplored for a long time. Recent publications have described a greater risk of developing osteonecrosis associated with bisphosphonate therapy and dental surgery. ${ }^{46-49}$ The pathophysiology behind the process has not been completely elucidated, but it is probably multifactorial and related to an alteration in the bone homeostasis and inhibition of angiogenesis. ${ }^{4-51}$ Because surgical intervention seems to be obligatory in the development of the majority of the osteonecrosis cases, ${ }^{47,48}$ it is conceivable that, in the present study, the combination of creating a bone defect and applying alendronate also evoked a negative bone response.
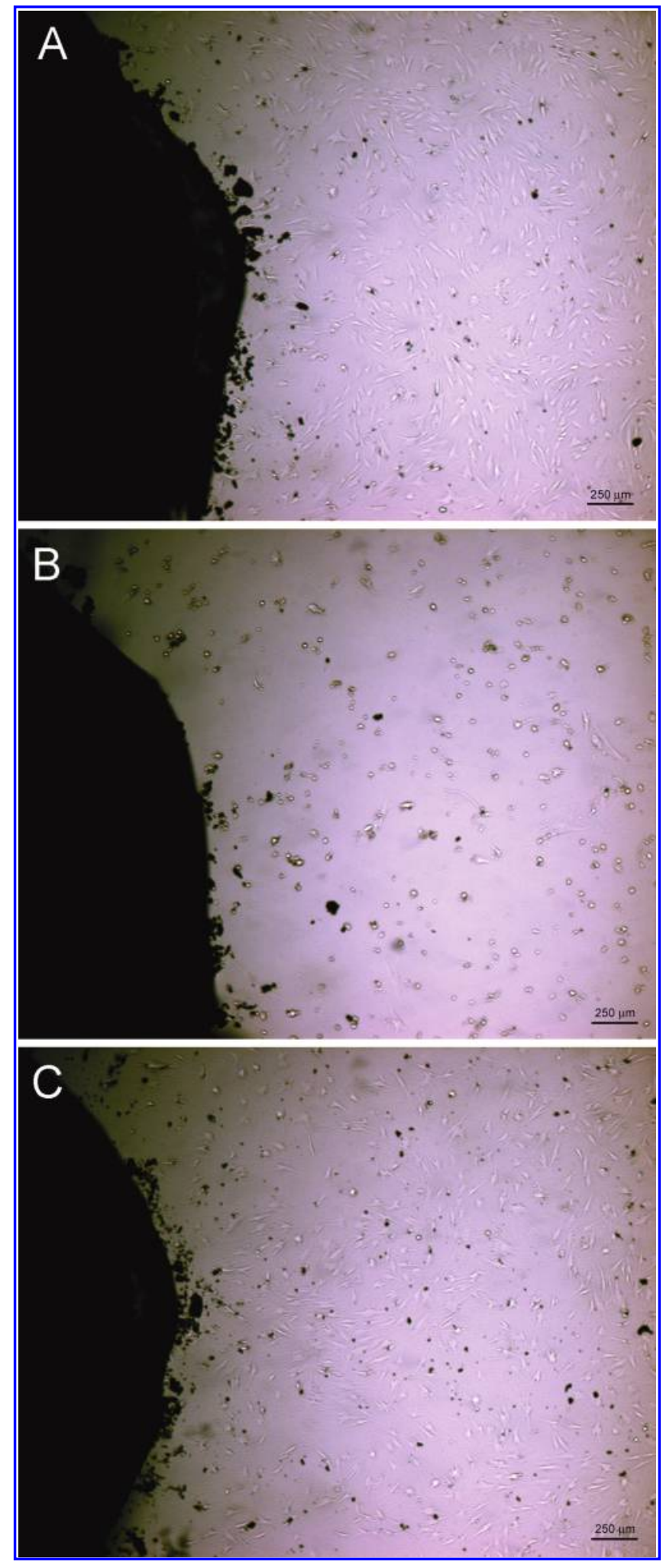

FIG. 7. The results of a cytotoxicity assay showing fibroblastic cells surrounding (A) plain, (B) alendronate, and (C) omeprazole bioactive bone cements after $72 \mathrm{~h}$ of incubation. Cell death was seen in the alendronate group. Color images available online at www.liebertpub.com/ten. 
In addition to alendronate, omeprazole was investigated as a possible bone antiresorptive agent in the G2B1 cement composites. In the clinic, omeprazole is used to treat dyspeptic problems, because it inhibits gastric acid secretion by targeting the gastric acid pump $\mathrm{H}+, \mathrm{K}(+)$-ATPase of the parietal cell membrane. ${ }^{19}$ Osteoclastic bone resorption is also regulated via a proton pump but of a different type than with gastric acid secretion. This vacuolar-type $\mathrm{H}+$ -ATPase generates acidity in the ruffled border zone of the osteoclast, which is responsible for bone resorption. ${ }^{25,26}$ In the present study, it was hypothesized that topically applied omeprazole could inhibit the osteoclastic proton pump, leading to greater bone formation in G2B1 cement composites in rabbits, because previous in vitro studies showed that this gastric PPI could affect osteoclast activity, ${ }^{20,21,23,24}$ but omeprazole did not influence BV or BMD around the implanted material, and bone contact was significantly lower in the omeprazole group than in plain controls after 6 weeks and was similar after 12 weeks of implantation. This observation may be associated with the finding that osteoclastic proton pumps are not sensitive for gastric PPIs and require high doses of omeprazole to be suppressed. ${ }^{20,24}$ In addition, to become activated, omeprazole first needs to be converted into an active inhibitor in an acidic environment. ${ }^{20,22,52}$ Tutunji et al. ${ }^{52}$ showed, using electroanalytical techniques, that in a solution buffered to $\mathrm{pH}$ values between 5.0 and 8.0, omeprazole degradation was significantly slower than more acidic solutions with a $\mathrm{pH}$ of 2.0 to 4.0. The secretory canaliculi of the gastric parietal cells have a $\mathrm{pH}$ of 1.0 or less, ${ }^{52}$ whereas resorption lacunae of bone have a $\mathrm{pH}$ of 4.5 to $6,{ }^{20,26}$ which theoretically can hamper the initial omeprazole conversion. In the present study, these above-described factors of limited omeprazole availability and the inability of activation due to unfavorable acidity might have played a role in the suggested hindered osteoclast inhibition as found in the omeprazole group.

In addition to experimental animal studies, a few clinical investigations have been performed on the topic of influencing bone formation with omeprazole treatment and also showed contradictory results. Kocsis et al. ${ }^{53}$ did not find different biochemical parameters of bone turnover in pediatric patients after omeprazole administration, which is in line with our results. On the other hand, a favorable bone response of omeprazole administration was seen in the study of Mizunashi et al., ${ }^{22}$ who showed a suppression in bone resorption in adult patients treated with gastric PPIs.

Although the current study did not find positive effects of local administration of alendronate or omeprazole, the study design might explain this. As mentioned earlier, a limitation of the present study was the lack of in vivo release data. Comparing the $\mu \mathrm{CT}$ results, BV surrounding the omeprazole implants was significantly lower after 12 weeks than after 6 weeks of implantation, and a similar trend was seen for the alendronate implants. In addition, the plain implants showed a trend toward less BV than with alendronate or omeprazole after 6 weeks of implantation. It can be speculated that both drugs had an early (burst) release profile leading to an increase in bone formation in the first weeks and diminished bone response after 12 weeks of implantation. Future studies should focus on the in vivo release profiles of both pharmaceuticals and their dose responses to further examine the possibility of topical instead of systemic application to provide bone-regenerative biomaterials with targeted antiresorptive agents.

\section{CONCLUSION}

Plain G2B1 bone cement composites showed good bone biological response in rabbit trabecular defects. Alendronate and omeprazole as applied to the cement composites in the current study design did not improve the bone response. Moreover, the released alendronate might have evoked a toxic reaction.

\section{ACKNOWLEDGMENTS}

This study was financially supported by DePuy CMW. The authors would like to thank Ms. M. van der Zande for her assistance with the cytotoxicity assay.

\section{REFERENCES}

1. Stains, J.P. and Civitelli, R. Cell-to-cell interactions in bone. Biochem Biophys Res Commun 328, 721, 2005.

2. Martin, T.J. and Sims, N.A. Osteoclast-derived activity in the coupling of bone formation to resorption. Trends 1 Med 11, 76, 2005.

3. Katagiri, T. and Takahashi, N. Regulatory mechanisms of osteoblast and osteoclast differentiation. Oral Dis 8, 147, 2002.

4. Raisz, L.G. Physiology and pathophysiology of bone remodeling. Clin Chem 45, 1353, 1999.

5. Kenny, S.M. and Buggy, M. Bone cements and fillers: a review. J Mater Sci Mater Med 14, 923, 2003.

6. Heini, P.F. and Berlemann, U. Bone substitutes in vertebroplasty. Eur Spine J 10 Suppl 2, 205, 2001.

7. Larsson, S. and Bauer, T.W. Use of injectable calcium phosphate cement for fracture fixation: a review. Clin Orthop Relat Res 395, 23, 2002.

8. Wozney, J.M. Overview of bone morphogenetic proteins. Spine 27, S2, 2002.

9. Jansen, J.A., Vehof, J.W., Ruhe, P.Q., Kroeze-Deutman, H., Kuboki, Y., Takita, H., Hedberg, E.L. and Mikos, A.G. Growth factor-loaded scaffolds for bone engineering. J Control Release 101, 127, 2005.

10. van Beek, E.R., Cohen, L.H., Leroy, I.M, Ebetino, F.H., Lowik, C.W., and Papapoulos, S.E. Differentiating the mechanisms of antiresorptive action of nitrogen containing bisphosphonates. Bone 33, 805, 2003.

11. Russell, R.G. Bisphosphonates: from bench to bedside. Ann N Y Acad Sci 1068, 367, 2006. 
12. McLeod, K., Anderson, G.I., Dutta, N.K., Smart, R.St.C., Voelcker, N.H., Sekel, R., and Kumar, S. Adsorption of bisphosphonate onto hydroxyapatite using a novel co-precipitation technique for bone growth enhancement. J Biomed Mater Res A 79, 271, 2006.

13. Jakobsen, T., Kold, S., Bechtold, J.E., Elmengaard, B., and Soballe, K. Local alendronate increases fixation of implants inserted with bone compaction: 12 -week canine study. J Orthop Res 25, 432, 2007.

14. Jakobsen, T., Kold, S., Bechtold, J.E., Elmengaard, B., and Soballe, K. Effect of topical alendronate treatment on fixation of implants inserted with bone compaction. Clin Orthop Relat Res 444, 229, 2006.

15. Tanzer, M., Karabasz, D., Krygier, J.J., Cohen, R., and Bobyn, J.D. The Otto Aufranc Award: bone augmentation around and within porous implants by local bisphosphonate elution. Clin Orthop Relat Res 441, 30, 2005.

16. Peter, B., Pioletti, D.P., Laib, S., Bujoli, B., Pilet, P., Janvier, P., Guicheux, J., Zambelli, P.-Y., Bouler, J.-M., and Gauthier, O. Calcium phosphate drug delivery system: influence of local zoledronate release on bone implant osteointegration. Bone 36, 52, 2005.

17. Meraw, S.J. and Reeve, C.M. Qualitative analysis of peripheral peri-implant bone and influence of alendronate sodium on early bone regeneration. J Periodontol 70, 1228, 1999.

18. Meraw, S.J., Reeve, C.M., and Wollan, P.C. Use of alendronate in peri-implant defect regeneration. J Periodontol 70, $151,1999$.

19. Horn, J. The proton-pump inhibitors: similarities and differences. Clin Ther 22, 266, 2000.

20. Mattsson, J.P., Vaananen, K., Wallmark, B., and Lorentzon, P. Omeprazole and bafilomycin, two proton pump inhibitors: differentiation of their effects on gastric, kidney and bone $\mathrm{H}(+)$-translocating ATPases. Biochim Biophys Acta 1065, 261,1991 .

21. Zaidi, M. Modularity of osteoclast behaviour and "modespecific" inhibition of osteoclast function. Biosci Rep 10, 547, 1990.

22. Mizunashi, K., Furukawa, Y., Katano, K., and Abe, K. Effect of omeprazole, an inhibitor of $\mathrm{H}+, \mathrm{K}(+)$-ATPase, on bone resorption in humans. Calcif Tissue Int 53, 21, 1993.

23. Tuukkanen, J. and Vaanane, H.K. Omeprazole, a specific inhibitor of $\mathrm{H}+-\mathrm{K}+-\mathrm{ATPase}$, inhibits bone resorption in vitro. Calcif Tissue Int 38, 123, 1986.

24. Hall, T.J. and Chambers, T.J. Na+/H+ antiporter is the primary proton transport system used by osteoclasts during bone resorption. J Cell Physiol 142, 420, 1990.

25. Rousselle, A.V. and Heymann, D. Osteoclastic acidification pathways during bone resorption. Bone 30, 533, 2002.

26. Vaes, G. Cellular biology and biochemical mechanism of bone resorption. A review of recent developments on the formation, activation, and mode of action of osteoclasts. Clin Orthop Relat Res 231, 239, 1988.

27. Goto, K., Shinzato, S., Fujibayashi, S., Tamura, J., Kawanabe, K., Hasegawa, S., Kowalski, R., and Nakamura, T. The biocompatibility and osteoconductivity of a cement containing beta-TCP for use in vertebroplasty. J Biomed Mater Res A 78, 629, 2006.

28. Aspenberg, P. Drugs and fracture repair. Acta Orthop 76, 741, 2005.
29. Bourne, M.H. Analgesics for orthopedic postoperative pain. Am J Orthop 33, 128, 2004.

30. Ooms, E.M., Wolke, J.G., van der Waerden, J.P., and Jansen, J.A. Trabecular bone response to injectable calcium phosphate (Ca-P) cement. J Biomed Mater Res 61, 9, 2002.

31. Fini, M., Giavaresi, G., Aldini, N.N., Torricelli, P., Botter, R., Beruto, D., and Giardino, R. A bone substitute composed of polymethylmethacrylate and alpha-tricalcium phosphate: results in terms of osteoblast function and bone tissue formation. Biomaterials 23, 4523, 2002.

32. Collinge, C., Merk, B., and Lautenschlager, E.P. Mechanical evaluation of fracture fixation augmented with tricalcium phosphate bone cement in a porous osteoporotic cancellous bone model. J Orthop Trauma 21, 124, 2007.

33. Verlaan, J. J., Oner, F. C., Slootweg, P. J., Verbout, A. J., and Dhert, W. J. Histologic changes after vertebroplasty. J Bone Joint Surg Am 86-A, 1230, 2004.

34. Lewis, G., Janna, S., and Bhattaram, A. Influence of the method of blending and antibiotic powder with an acrylic bone cement powder on physical, mechanical, and thermal properties of the cured cement. Biomaterials 26, 4317, 2005.

35. Lewis, G. and Janna, S. Alendronate in bone cement. Clin Orthop Reat Res 445, 233, 2006.

36. Ruhe, P.Q., Boerman, O.C., Russell, F.G.M., Spauwen, P.H.M., Mikos, A.G., and Jansen, J.A. Controlled release of rhBMP-2 loaded poly(DL-lactic-co-glycolic acid)/calcium phosphate cement composites in vivo. J Control Rel, 106, 162, 2005.

37. Lewis $\mathrm{G}$. and Janna $\mathrm{S}$. The in vitro evaluation of gentamycin sulfate form a commercially available gentamycin-loaded acrylic bone cement, VersaBond TM AB. J Biomed Mater Res Part B Appl Biomater 71B, 77, 2004.

38. Astrand, J. and Aspenberg, P. Topical, single dose bisphosphonate treatment reduced bone resorption in a rat model for prosthetic loosening. J Orthop Res 22, 244, 2004.

39. Binderman, I., Adut, M., and Yaffe, A. Effectiveness of local delivery of alendronate in reducing alveolar bone loss following periodontal surgery in rats. J Periodontol 71, 1236, 2000.

40. Aspenberg, P. and Astrand, J. Bone allografts pretreated with a bisphosphonate are not resorbed. Acta Orthop Scand 73, 20, 2002.

41. Denissen, H., Montanari, C., Martinetti, R., van Lingen, A., and van den Hooff, A. Alveolar bone response to submerged bisphosphonate-complexed hydroxyapatite implants. J Periodontol 71, 279, 2000.

42. Lehman, R.A. Jr, Kuklo, T.R., Freedman, B.A., Cowart, J.R., Mense, M.G., and Riew, K.D. The effect of alendronate sodium on spinal fusion: a rabbit model. Spine J 4, 36, 2004.

43. Sama, A.A., Khan, S.N., Myers, E.R., Huang, R.C., Cammisa, F.P., Sandhu, H.S., and Lane, J.M. High-dose alendronate uncouples osteoclast and osteoblast function: a study in a rat spine pseudarthrosis model. Clin Orthop Relat Res 425, 135 , 2004.

44. Marshall, J.K. The gastrointestinal tolerability and safety of oral bisphosphonates. Expert Opin Drug Saf 1, 71, 2002.

45. Suri, S., Monkkonen, J., Taskinen, M., Pesonen, J., Blank, M.A., Phipps, R.J., and Rogers, M.J. Nitrogencontaining bisphosphonates induce apoptosis of Caco-2 cells in vitro by inhibiting the mevalonate pathway: a model of 
bisphosphonate-induced gastrointestinal toxicity. Bone 29, 336, 2001.

46. Carter, G., Goss, A.N., and Doecke, C. Bisphosphonates and avascular necrosis of the jaw: a possible association. Med J Aust 182, 413, 2005.

47. Melo, M.D. and Obeid, G. Osteonecrosis of the jaws in patients with a history of receiving bisphosphonate therapy: strategies for prevention and early recognition. J Am Dent Assoc 136, 1675, 2005.

48. Dimitrakopoulos, I., Magopoulos, C., and Karakasis, D. Bisphosphonate-induced avascular osteonecrosis of the jaws: a clinical report of 11 cases. Int J Oral Maxillofac Surg 35, 588, 2006.

49. Ruggiero, S.L., Mehrotra, B., Rosenberg, T.J., and Engroff, S.L. Osteonecrosis of the jaws associated with the use of bisphosphonates: a review of 63 cases. J Oral Maxillofac Surg 62, 527, 2004.

50. Migliorati, C.A., Schubert, M.M., Peterson, D.E., and Seneda, L.M. Bisphosphonate-associated osteonecrosis of mandibular and maxillary bone. Cancer 104, 83, 2005.

51. Hasmim, M., Bieler, G., and Ruegg, C. Zoledronate inhibits endothelial cell adhesion, migration and survival through the suppression of multiple, prenylation-dependent signaling pathways. J Thromb Haemost 5, 166, 2007.

52. Tutunji, M.F., Qaisi, A.M., El-Eswed, B.I., and Tutunji, L.F. Reactions of sulfenic acid with 2-mercaptoethanol: a mechanism for the inhibition of gastric $(\mathrm{H}+-\mathrm{K}+)$-adenosine triphosphate by omeprazole. J Pharm Sci 96, 196, 2007.

53. Kocsis, I., Arato, A., Bodanszky, H., Szonyi, L., Szabo, A., Tulassay, T., and Vasarhelyi, B. Short-term omeprazole treatment does not influence biochemical parameters of bone turnover in children. Calcif Tissue Int 71, 129, 2002.

Address reprint requests to: John A. Jansen, D.D.S., Ph.D. Professor

Department of Periodontology and Biomaterials Radboud University Nijmegen Medical Center P.O. Box 9101, 6500 HB Nijmegen The Netherlands

E-mail: j.jansen@dent.umcn.nl 



\section{This article has been cited by:}

1. J.J. Verlaan, M.A. Lopez-Heredia, J. Alblas, F.C. Oner, J.A. Jansen, W.J.A. DhertInjectable Bone Cements for Spinal Column Augmentation: Materials for Kyphoplasty/Vertebroplasty 147-160. [CrossRef]

2. Jessica A. Cottrell, Francis M. Vales, Deborah Schachter, Scott Wadsworth, Rama Gundlapalli, Rasesh Kapadia, J. Patrick O'Connor. 2010. Osteogenic Activity of Locally Applied Small Molecule Drugs in a Rat Femur Defect Model. Journal of Biomedicine and Biotechnology 2010, 1-12. [CrossRef]

3. Seung-Jun Ku, Young-Il Chang, Chang-Hoon Chae, Seong-Gon Kim, Young-Wook Park, Youn-Kwan Jung, Je-Yong Choi. 2009. Static tensional forces increase osteogenic gene expression in three-dimensional periodontal ligament cell culture. $B M B$

Reports 42:7, 427-432. [CrossRef] 